\title{
Expression of FRS2 in atypical lipomatous tumor/well-differentiated liposarcoma and dedifferentiated liposarcoma: an immunohistochemical analysis of 182 cases with genetic data
}

Wenyi Jing ${ }^{1}$, Ting Lan ${ }^{2}$, Yan Qiu ${ }^{1}$, Ran Peng ${ }^{1}$, Yang Lu ${ }^{1}$ Huijiao Chen ${ }^{1}$, Min Chen ${ }^{1}$, Xin He${ }^{1}$, Chen Chen ${ }^{1}$ and Hongying Zhang ${ }^{1 *}$

\begin{abstract}
Background: The fibroblast growth factor receptor substrate 2 (FRS2) gene is located close to MDM2 and CDK4 within the 12q13-15 chromosomal region. FRS2 gene was recently found to be consistently amplified in atypical lipomatous tumor (ALT)/well-differentiated liposarcoma (WDL) and dedifferentiated liposarcoma (DDL), suggesting the detection of FRS2 amplification could be a diagnostic tool for ALT/WDL/DDLs. However, the expression of FRS2 protein and diagnostic value of FRS2 immunohistochemistry (IHC) has not been evaluated in a large cohort of ALT/ WDL/DDLS.
\end{abstract}

Methods: A SNOMED search of hospital surgical pathology files from January 2007 to July 2020 identified 182 ALT/ WDL/DDLs with available materials. FRS2 fluorescence in situ hybridization (FISH) and IHC were performed on 182 ALT/WDL/DDLs and 64 control samples. The expression of FRS2 was also compared with that of classic immunomarkers (MDM2 and CDK4) of this tumor entity.

Results: This study included 91 ALT/WDLs and 91 DDLs. The FISH results showed 172 of 182 (94.5\%) cases were FRS2-amplified, and 10 cases were FRS2-nonamplified. Immunostaining results showed 171 (94.0\%) ALT/WDL/DDLs were positive for FRS2 and 11 cases (6.0\%) were FRS2-immunonegative. In 172 FRS2-amplified cases, 166 (96.5\%) were FRS2-immunopositive, and 6 (3.5\%) were negative. Among 10 FRS2-nonamplified ALT/WDL/DDL cases, 5 cases were FRS2-immunonegative, and 5 tumors displayed 1+ staining for this marker. In 64 control cases, none of them exhibited FRS2 amplification. Forty-seven (73.5\%) control cases were negative for FRS2 immunostaining, while 17 cases (26.5\%) were FRS2-immunopositive. Fifteen of these false positive samples (15/17, 88.2\%) showed 1+ positivity and only 2 cases $(2 / 17,11.8 \%)$ displayed $2+$ positivity. In ALTMDL/DDLs, the sensitivity of FRS2 immunostaining was slightly lower than MDM2 (FRS2 vs. MDM2: 94.0\% vs 100.0\%) and CDK4 (FRS2 vs. CDK4: $94.0 \%$ vs 97.0\%). However, the specificity of FRS2 (73.5\%) was slightly higher than that of MDM2 (67.8\%) and CDK4 (64.4\%).

\footnotetext{
* Correspondence: hy_zhang@scu.edu.cn

'Department of Pathology, West China Hospital, Sichuan University, Guoxuexiang 37, Chengdu 610041, Sichuan, China

Full list of author information is available at the end of the article
} changes were made. The images or other third party material in this article are included in the article's Creative Commons licence, unless indicated otherwise in a credit line to the material. If material is not included in the article's Creative Commons licence and your intended use is not permitted by statutory regulation or exceeds the permitted use, you will need to obtain permission directly from the copyright holder. To view a copy of this licence, visit http://creativecommons.org/licenses/by/4.0/ The Creative Commons Public Domain Dedication waiver (http://creativecommons.org/publicdomain/zero/1.0/) applies to the data made available in this article, unless otherwise stated in a credit line to the data. 
Conclusion: This study indicated that FRS2 IHC had relatively good consistency with FRS2 FISH, suggesting that FRS2 immunostaining could be utilized as an additional screening tool for the diagnosis of ALT/WDL/DDL. It must be emphasized that MDM2/CDK4/FRS2 especially MDM2 FISH remains the gold standard and the most recommended method to diagnose this entity.

Keywords: Atypical lipomatous tumor, Well-differentiated liposarcoma, Dedifferentiated liposarcoma, Immunohistochemistry, FRS2

\section{Background}

Liposarcoma is one of the most common soft tissue sarcomas in adults and consists of four subtypes: atypical lipomatous tumor (ALT)/well-differentiated liposarcoma (WDL)/dedifferentiated liposarcoma (DDL), myxoid liposarcoma, pleomorphic liposarcoma, and myxoid pleomorphic liposarcoma [1-3]. The ALT/WDL/DDL subgroup accounts for the majority of liposarcomas, exhibiting amplification of the chromosome 12q13-15 region, leading to the amplification of $M D M 2, C D K 4$, HMGA2 and CPM [4-6]. Hence, fluorescence in situ hybridization (FISH) for the identification of MDM2/ CDK4 amplification or immunohistochemistry (IHC) for the detection of their overexpression were recommended as useful ancillary tools for the diagnosis of ALT/WDL/ DDL [7-13].

The fibroblast growth factor receptor substrate 2 (FRS2) gene is also located in the 12q13-15 region close to the MDM2 and CDK4 genes. In 2011, Wang et al. reported the consistent amplification of the FRS2 gene in ALT/WDL/DDLs (57 of 57, 100\%) [14]. Later, Zhang et al. verified FRS2 amplification in 15 DDLs, with an amplification frequency of 93\% (14/15) [15]. Our recent work also identified that the FRS2 gene is highly amplified in 146 ALT/WDL/DDL cases (136/146, 93.2\%) [16]. These results indicated that FRS2 have diagnostic utility for this tumor group. Because the lack of commercial FRS2 FISH probes and the evaluation of FISH requires specific equipment and experienced cytogeneticists. Furthermore, IHC is a more economical alternative and could be carried out in most hospitals in clinical practice. Hence, it is necessary to examine the utility of FRS2 IHC for the diagnosis of ALT/WDL/DDL. Moreover, FRS2 acts as a key adaptor protein in the fibroblast growth factor receptor (FGFR) pathway, subsequently activating downstream signaling pathways [17]. Hence, the detection of FRS2 protein expression in ALT/WDL/ DDL may also provide clues for their therapy in the future. However, the expression of FRS2 in ALT/WDL/ DDL and the utility of FRS2 IHC for the differential diagnosis of this disease spectrum have not been widely investigated. FRS2 IHC was only performed by Zhang et al. in 11 DDLs, among which $9(82 \%)$ cases were positive for FRS2 [15].
Here, we performed FRS2 immunostaining in 182 ALT/WDL/DDL and 64 control cases, and the results were compared with FRS2 FISH data of the 182 cases to evaluate the sensitivity, specificity and diagnostic value of FRS2 IHC for this entity. Moreover, the MDM2 and CDK4 IHC results of most cases were reviewed to compare FRS2 and these classic immunomarkers for ALT/ WDL/DDL.

\section{Methods \\ Case selection}

This study was approved by the West China Hospital Institutional Review Board. A SNOMED search of the hospital surgical pathology files from January 2007 to July 2020 identified 182 ALT/WDL/DDLs with available materials for further study. Histological sections as well as previous MDM2 and CDK4 IHC slides of the tumors were reviewed independently by two pathologists with soft tissue tumor pathology expertise (H.Z. and H.C.) and 2 general surgical pathologists (W.J. and T.L.) independently. Classification was performed according to the World Health Organization criteria, and grading was carried out following the 'modified' Federation Nationale des Centres de Lutte Contre le Cancer (FNCLCC) grading system [2, 18]. Clinicopathological information was collected from clinical records and pathology reports. The control cases were obtained independently from clinical cases, including conventional lipoma $(n=25)$, spindle cell lipoma $(n=7)$, atypical spindle cell lipomatous tumor $(n=3)$, myxoid liposarcoma $(n=2)$, pleomorphic liposarcoma $(n=8)$, myxofibrosarcoma $(n=5)$, leiomyosarcoma $(n=5)$, schwannoma $(n=1)$, undifferentiated pleomorphic sarcoma $(n=3)$, osteosarcoma $(n=1)$, low-grade myofibroblastic sarcoma $(n=1)$, and normal fat $(n=3)$.

\section{Fluorescence in situ hybridization (FISH)}

FRS2 FISH was performed on 182 ALT/WDL/DDLs, among which 146 cases had archival FRS2 FISH records that were published previously [16], and additional FRS2 FISH analyses were performed on the remaining 36 cases. MDM2 FISH was performed on all FRS2 nonamplified ALT/WDL/DDL cases. FISH assays were carried out according to an established laboratory protocol $[8$, 19]. A bacterial artificial chromosome (BAC) clone for 
FRS2 (RP11-956E11) was purchased from the Children's Hospital Oakland Research Institute (CHORI, Oakland, CA, USA). Preparation and validation of the FRS2 probe was performed according to a previously published study [14]. A Vysis LSI MDM2 dual-color probe (Abbott Molecular, Des Plaines, IL, USA) was used for the MDM2 FISH assay. Tumors were scored by two investigators counting 100 nuclei in a blinded fashion. The hemorrhage and necrotic areas were excluded as much as possible when making the evaluation. The amplification of $F R S 2$ or MDM2 was defined as FRS2/CEP12 or $M D M 2 / C E P 12 \geq 2.0$, while a ratio $<2.0$ was considered nonamplified and a ratio $<2.0$ with more than two signals was considered polysomic for CEP12.

\section{Immunohistochemistry (IHC)}

Immunohistochemical staining was performed on the representative sections of each case by FRS2 antibody (clone SC-8318, Santa Cruz Biotechnology, USA), MDM2 antibody (clone SMP14, ready-to-use; Dako, Carpinteria, CA) and CDK4 antibody (clone EP180, 1: 100; Dako, Carpinteria, CA), using the EnVision Plus detection system (DAKO, Carpinteria CA, USA). Sections were revealed by diaminobenzidine solution (Dako, Carpintera CA, USA) and counterstained with hematoxylin. Positive and negative control sections were utilized. The results were evaluated independently by pathologists (H.Z., T.L. and Y.Q.) using the same standard, and the hemorrhage and necrotic areas were excluded as much as possible when making the evaluation. Regarding FRS2, cytoplasmic staining was considered positive, whereas nuclear staining for MDM2 and CDK4 was recorded as positive staining. IHC staining intensity was scored as none (0), weak (1), moderate (2), or strong (3), and the extent of positive tumor cells was scored as < $5 \%$ (0), $5-10 \%$ (1), $11-49 \%$ (2), $50-74 \%$ (3), or $\geq 75 \%$ (4). Cases were graded as negative - (0-1), weakly positive $1+(2-4)$, moderately positive $2+(6-8)$, and strongly positive $3+(9-12)$ by multiplying the two scores [20].

\section{Statistical analysis}

The sensitivity and specificity of FRS2 IHC were evaluated in the ALT/WDL and DDL subgroups to assess the ability of the immunomarker to correctly classify ALT/ WDL/DDL from their histologic mimics. Student's $t$-test was used to compare continuous variables, and $\mathrm{McNe}$ mar and kappa tests were utilized to evaluate the agreement between FRS2 FISH and FRS2 IHC. The chisquare test was used to compare the positive expression rates of FRS2 with the other immunohistochemical markers by SPSS version 20.0 (IBM Corp, Armonk, NY, USA). $P$-values $<0.05$ were considered significant.

\section{Results}

\section{Clinical and pathological findings}

This study included 108 males and 74 females aged 2083 years (median: 57 years). Tumors ranged in size from 1.2 to $45.0 \mathrm{~cm}$ (median: $16.0 \mathrm{~cm}$ ). The majority of tumors were located in the retroperitoneum $(n=92)$, followed by the extremities $(n=36)$, abdominopelvic cavity $(n=28)$, trunk $(n=10)$, head and neck area $(n=$ $8)$, scrotum/inguinal region $(n=6)$ and mediastinum $(n=2)$. The study contained 91 ALT/WDLs and 91 DDLs. The 91 ALT/WDL cases were graded into FNCL CC 1, composed of 48 lipoma-like (52.7\%), 40 sclerotic (44.0\%), and 3 inflammatory subtypes (3.3\%). In the 91 DDLs, the dedifferentiated components had a variety of morphologic patterns. Sixty-six DDLs manifested as myxofibrosarcoma/fibrosarcoma $(n=53,58.2 \%)$ and undifferentiated pleomorphic sarcoma $(n=13,14.3 \%)$. Six tumors (6.6\%) had low-grade fibrosarcoma-like differentiation areas. Heterologous differentiation $(n=11,12.1 \%)$ and homologous pleomorphic liposarcoma-like differentiation exhibited in 8 DDLs (8.8\%). Grading results showed that 39 (42.9\%) DDLs were graded into FNCL CC 2, and 52 (57.1\%) tumors were FNCLCC 3.

\section{FRS2 FISH analysis}

FRS2 amplification was identified in 172 of 182 (94.5\%) cases, including 84 ALT/WDLs (84/91, 92.3\%) and 88 DDLs (88/91, 96.7\%). The 10 FRS2 non-amplified cases were $M D M 2$ amplified, including 7 ALT/WDLs and 3 DDLs. None of the control samples $(n=64)$ showed amplification of FRS2.

\section{FRS2 immunostaining results and the comparison with FRS2 FISH results} IHC results of $A L T / W D L / D D L s$ and control cases

The immunohistochemical results are summarized in Table 1 . FRS2 cytoplasmic positivity was observed in 171/ 182 (94.0\%) ALT/WDL/DDL cases (Figs. 1 and 2), among which 57 cases had $3+$ staining (31.3\%), 63 had 2+ staining (34.7\%), 51 (28.0\%) tumors displayed $1+$ staining and 11 cases (6.0\%) were FRS2-negative. In 91 ALT/WDL cases, $83(91.2 \%)$ cases were positive for FRS2, including 50 (54.9\%) ALT/WDLs with 2+/3+ staining, 33 (36.3\%) cases with $1+$ positivity and the remaining $8(8.8 \%)$ cases were negative for FRS2. In terms of DDLs, 88 (96.7\%) tumors were FRS2 positive, containing 18 (19.8\%) cases with $1+$ positivity and $70(76.9 \%)$ tumors with $2+/ 3+$ staining, and only 3 (3.3\%) DDL cases were FRS2 negative.

In 64 control samples, 47 (73.5\%) cases were negative, and 17 (26.5\%) cases demonstrated FRS2 positivity (Fig. 3). Fifteen of these 17 false positive cases (88.2\%) displayed 1+ staining, including 5 lipomas, 2 spindle cell lipomas, 2 pleomorphic liposarcomas, 1 myxoid liposarcoma, 1 atypical spindle cell lipomatous tumor, 1 undifferentiated 
Table 1 FRS2 immunostaining in 182 ALT/WDL/DDLs and 64 control cases

\begin{tabular}{|c|c|c|c|c|c|}
\hline \multirow[t]{2}{*}{ Tumor type } & \multicolumn{4}{|c|}{ FRS2 immunostaining } & \multirow[t]{2}{*}{ Total } \\
\hline & $-(\%)$ & $1+(\%)$ & $2+(\%)$ & $3+(\%)$ & \\
\hline ALT/WDL/DDL & $11(6.0 \%)$ & $51(28.0 \%)$ & $63(34.7 \%)$ & $57(31.3 \%)$ & 182 \\
\hline ALT/WDL & $8(8.8 \%)$ & $33(36.3 \%)$ & $31(34.1 \%)$ & $19(20.8 \%)$ & 91 \\
\hline $\mathrm{DDL}$ & $3(3.3 \%)$ & $18(19.8 \%)$ & $32(35.2 \%)$ & $38(41.7 \%)$ & 91 \\
\hline Control group & $47(73.5 \%)$ & $15(23.4 \%)$ & $2(3.1 \%)$ & $0(0 \%)$ & 64 \\
\hline Normal adipose tissue & $3(100 \%)$ & $0(0 \%)$ & $0(0 \%)$ & $0(0 \%)$ & 3 \\
\hline \multicolumn{6}{|l|}{ Benign adipose tumor } \\
\hline Lipoma & $20(80.0 \%)$ & $5(20 \%)$ & $0(0 \%)$ & $0(0 \%)$ & 25 \\
\hline Spindle cell lipoma & $5(71.4 \%)$ & $2(28.6 \%)$ & $0(0 \%)$ & $0(0 \%)$ & 7 \\
\hline \multicolumn{6}{|l|}{ Other spindle cell tumors } \\
\hline Pleomorphic liposarcoma & $5(62.5 \%)$ & $2(25.0 \%)$ & $1(12.5 \%)$ & $0(0 \%)$ & 8 \\
\hline Myxoid liposarcoma & $1(50.0 \%)$ & $1(50.0 \%)$ & $0(0 \%)$ & $0(0 \%)$ & 2 \\
\hline Atypical spindle cell lipomatous tumor & $2(66.7 \%)$ & $1(33.3 \%)$ & $0(0 \%)$ & $0(0 \%)$ & 3 \\
\hline Undifferentiated pleomorphic sarcoma & $2(66.7 \%)$ & $1(33.3 \%)$ & $0(0 \%)$ & $0(0 \%)$ & 3 \\
\hline Myxofibrosarcoma & $5(100 \%)$ & $0(0 \%)$ & $0(0 \%)$ & $0(0 \%)$ & 5 \\
\hline Leiomyosarcoma & $3(60.0 \%)$ & $2(40.0 \%)$ & $0(0 \%)$ & $0(0 \%)$ & 5 \\
\hline Low-grade myofibroblastic sarcoma & $0(0 \%)$ & $0(0 \%)$ & $1(100 \%)$ & $0(0 \%)$ & 1 \\
\hline Osteosarcoma & $0(0 \%)$ & $1(100 \%)$ & $0(0 \%)$ & $0(0 \%)$ & 1 \\
\hline Schwannoma & $1(100 \%)$ & $0(0 \%)$ & $0(0 \%)$ & $0(0 \%)$ & 1 \\
\hline
\end{tabular}

$\overline{A L T / W D L}$ Atypical lipomatous tumor/well-differentiated liposarcoma, DDL Dedifferentiated liposarcoma, - negativity, $1+$ weak positivity, 2+ moderate positivity, 3+ strong positivity

pleomorphic sarcoma, 2 leiomyosarcomas and 1 osteosarcoma. Only 2 cases $(2 / 17,11.8 \%)$ displayed $2+$ staining, including 1 pleomorphic liposarcoma and 1 low-grade myofibroblastic sarcoma.

\section{Comparison of FRS2 IHC and FISH results in the ALT/ WDL/DDL}

Immunostaining results in FRS2-amplified cases

The comparison of FRS2 IHC and FISH results are summarized in Table 2. Among 172 FRS2-amplified cases (84 ALT/ WDLs, 88 DDLs), 166 cases (96.5\%) had consistent positive IHC results with their FISH results, while 6 (3.5\%) FRS2amplified cases were negative for FRS2 immunostaining. Statistical analysis found that FRS2 IHC had relatively good consistency with FRS2 FISH $(P<0.05$, kappa $=0.444)$. The 166 FRS2-immunopositive cases consisted of 57 cases (19 ALT/WDLs and 38 DDLs) with 3+ positivity, 63 (31ALT/ WDLs and 32 DDLs) with $2+$ positivity and 46 cases (31 ALT/WDLs and 15 DDLs) with 1+ staining. In the 6 FRS2immunonegative cases, 2 cases were samples from 5 years ago, and necrosis or hemorrhage was seen in 3 cases.

\section{Immunostaining results in FRS2-nonamplified cases}

Among 10 FRS2 gene-nonamplified cases (7 ALT/WDLs and 3 DDLs), the IHC results of 5 cases were in agreement with FISH results, showing negative FRS2 immunostaining. However, 5 cases were FRS2- immunopositive, with $1+$ positivity. The 5 FRS2immunopositive cases were composed of 2 ALT/WDLs and 3 DDLs, among which 2 cases had necrotic areas and 1 sample had hemorrhagic areas.

All 64 control samples were FRS2 nonamplified, while 17 of them (26.5\%) were FRS2-immunopositive. Two cases had $2+$ staining, including 1 low-grade myofibroblastic sarcoma and 1 pleomorphic liposarcoma, and the latter was polysomic for CEP12. In the 15 cases with $1+$ staining, 2 tumors also harbored a polysomic pattern (1 pleomorphic liposarcoma and 1 undifferentiated pleomorphic sarcoma). Other changes such as necrosis or hemorrhage were found in 2 leiomyosarcomas, 1 pleomorphic liposarcoma, 1 lipoma, and 1 spindle cell lipoma.

\section{Diagnostic values of FRS2 immunostaining for ALT/WDL/ DDL}

The diagnostic sensitivity and specificity of FRS2 IHC in distinguishing ALT/WDL/DDL from other histological mimics were 94.0 and $73.5 \%$, respectively (Table 3 ). With the standard of $2+$ staining, the specificity reached $96.9 \%$, while the sensitivity decreased to $66.0 \%$. The sensitivity and specificity of FRS2 IHC for distinguishing ALT/WDL from normal fat tissue and other benign adipose tumors (lipoma, spindle cell lipoma) were 91.2 and $80.0 \%$, and those of $2+$ FRS2 were 54.9 and $100.0 \%$, respectively. In the differentiation of DDL from other 

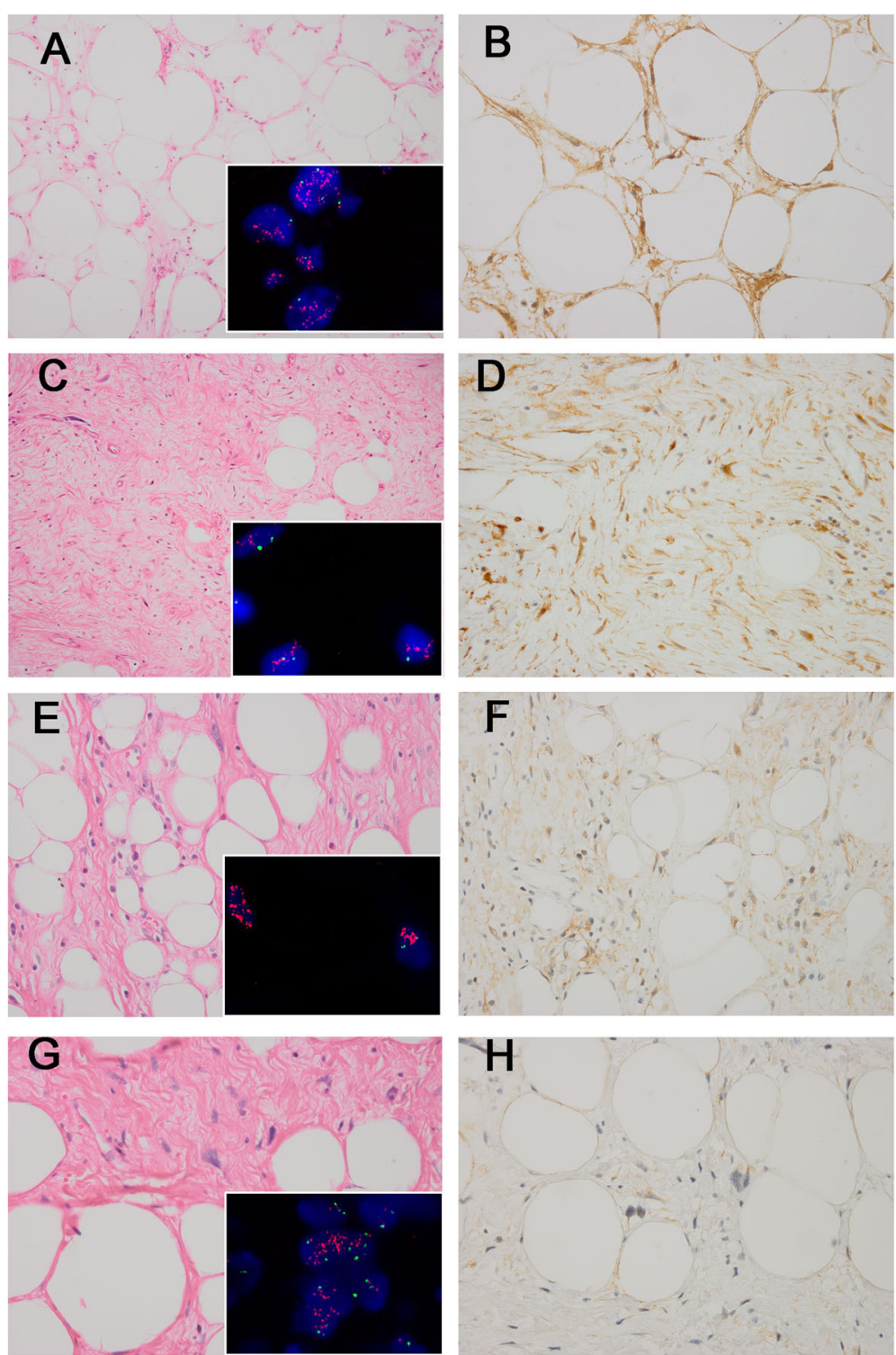

Fig. 1 The histologic features of ALT/WDLs and corresponding FRS2 FISH and FRS2 immunostaining results. Lipoma-like ALT/WDL(a) with FRS2 amplification (inset), showing 3+ FRS2 expression with diffuse and strong cytoplasmic staining (b). Sclerotic ALT/WDL(c) showing FRS2 amplification (inset), displayed 3+ FRS2 immunostaining, with strong FRS2 protein expression (d). Lipoma-like ALT/WDL(e) with FRS2 amplification (inset), exhibited 2+ cytoplasmic staining for FRS2 (f). Sclerosing ALTMDL (g) with FRS2 amplification (inset), showing 1+ FRS2 positivity (h). Red signals represent FRS2. Green signals represent chromosome 12 centromeres. (a, c, e, g, haematoxylin and eosin; b, d, f, h, immunostaining) (original magnification, a, c $\times 200, b, d-h \times 400$ )

spindle cell tumors, the sensitivity and specificity were 96.7 and $65.5 \%, 76.9$ and $93.1 \%$ by using $1+$ staining and $2+$ staining as cutoff values, respectively.

\section{Comparison of FRS2 IHC results with classic diagnostic IHC markers in ALT/WDL/DDL and control cases}

In our series, 177 tumors had prior MDM2 immunostaining results, and 164 had CDK4 results (Fig. 4). Among the 177 cases, all displayed MDM2 nuclear staining (100.0\%), including 86 ALT/WDLs and 91 DDLs, among which 98 cases $(55.4 \%)$ with $3+$ positivity, 52 cases $(29.4 \%)$ with $2+$ positivity and $27(15.2 \%)$ tumors displayed $1+$ staining. The CDK4 immunostaining results showed that 159 of 164 cases $(97.0 \%)$ were positive for CDK4, containing 77 ALT/WDLs and 82 DDLs, with 81 cases (49.4\%) showing $3+$ positivity, 43 cases $(26.2 \%)$ with $2+$ positivity and 35 cases (21.4\%) with $1+$ staining. In ALT/WDL/DDLs, the sensitivity of FRS2 immunostaining was generally similar to that of MDM2 (FRS2 vs. MDM2: $94.0 \%$ vs $100.0 \%$ ) and CDK4 (FRS2 vs. CDK4: $94.0 \%$ vs $97.0 \%$ ) and slightly lower 

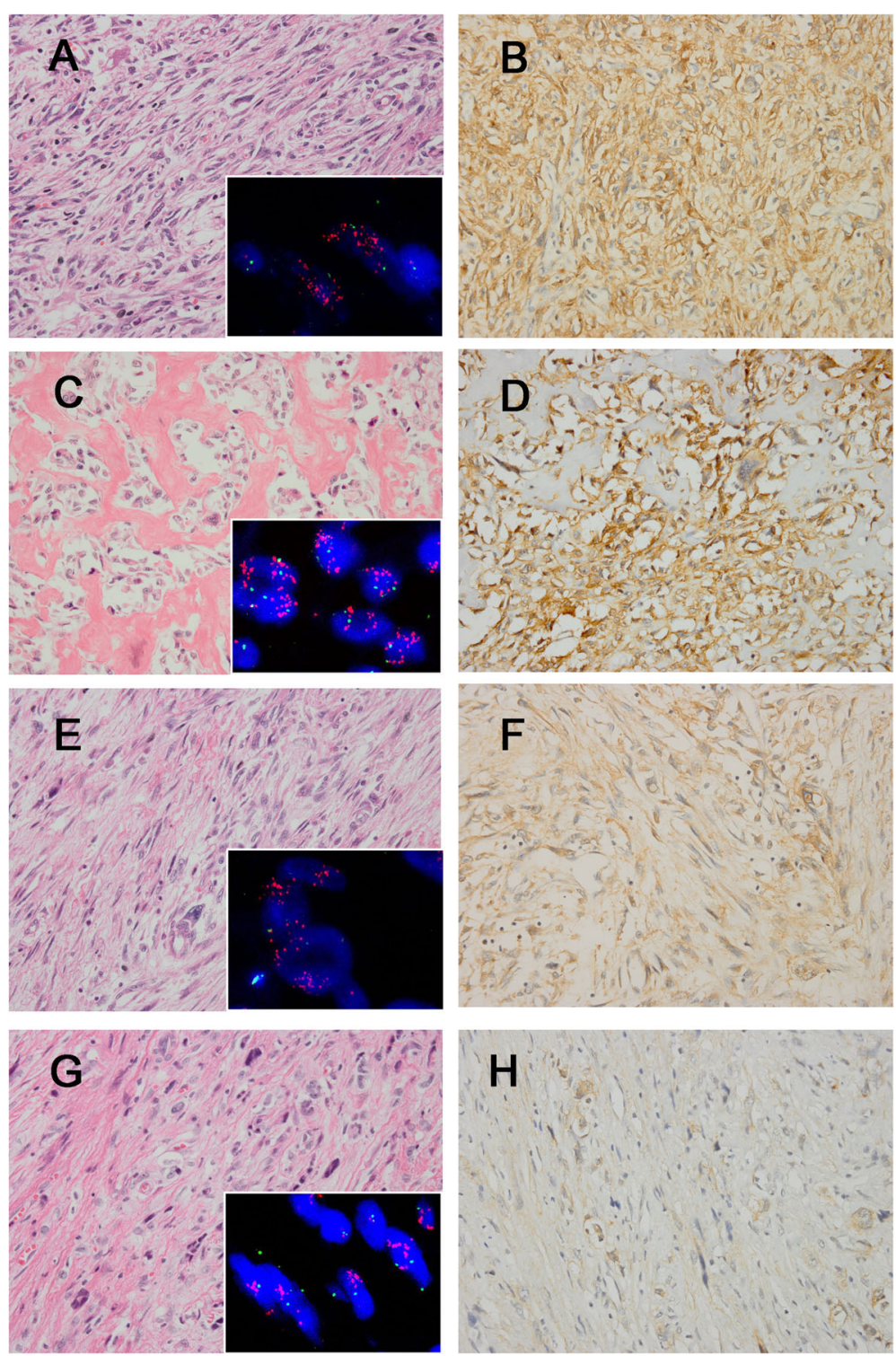

Fig. 2 The histologic features of DDLs and corresponding FRS2 FISH and FRS2 immunostaining results. DDL with fibrosarcoma-like differentiation(a), showing FRS2 amplification (inset), displayed 3+ FRS2 immunostaining, exhibiting diffuse and strong cytoplasmic FRS2 expression (b). DDL showing osteosarcoma-like differentiation(c), with FRS2 amplification (inset), had 3+ FRS2 staining, harboring strong FRS2 immunostaining (d). DDL demonstrating undifferentiated sarcoma-like differentiation(e), with FRS2 amplification (inset), harbored 2+ FRS2 staining (f). DDL with leiomyosarcoma-like differentiation(g) harboring FRS2 amplification (inset) showed 1+ positivity for FRS2 immunostaining (h). Red signals represent FRS2. Green signals represent chromosome12 centromeres. (a, c, e, g, haematoxylin and eosin; b, d, f, h, immunostaining) (original magnification $a-h \times 400)$

than these two classic immunomarkers. Moreover, FRS2 immunostaining was positive in 166 of 172 FRS2 geneamplified cases, with a consistency rate of $96.5 \%$, which was similar to the consistency rate of MDM2 immunostaining (100\%).

MDM2 and CDK4 IHC was performed in 59 control cases with available material. The MDM2 immunostaining result showed 40 cases $(67.8 \%)$ were negative for MDM2 and 19 were positive, including 16 displaying 1+ positivity and 3 with $2+$ positivity. The CDK 4 IHC result revealed 38 cases $(64.4 \%)$ were negative for CDK4 and 21 cases were positive, among which 17 cases had 1+ positivity and 4 cases showed $2+$ positivity. The specificities of MDM2 and CDK4 immunostaining were 67.8 and $64.4 \%$, respectively, slightly lower to that of FRS2 immunostaining which was $73.5 \%$.

\section{Discussion}

The diagnosis of ALT/WDL/DDL based only on histological features could sometimes be challenging. Owing to 

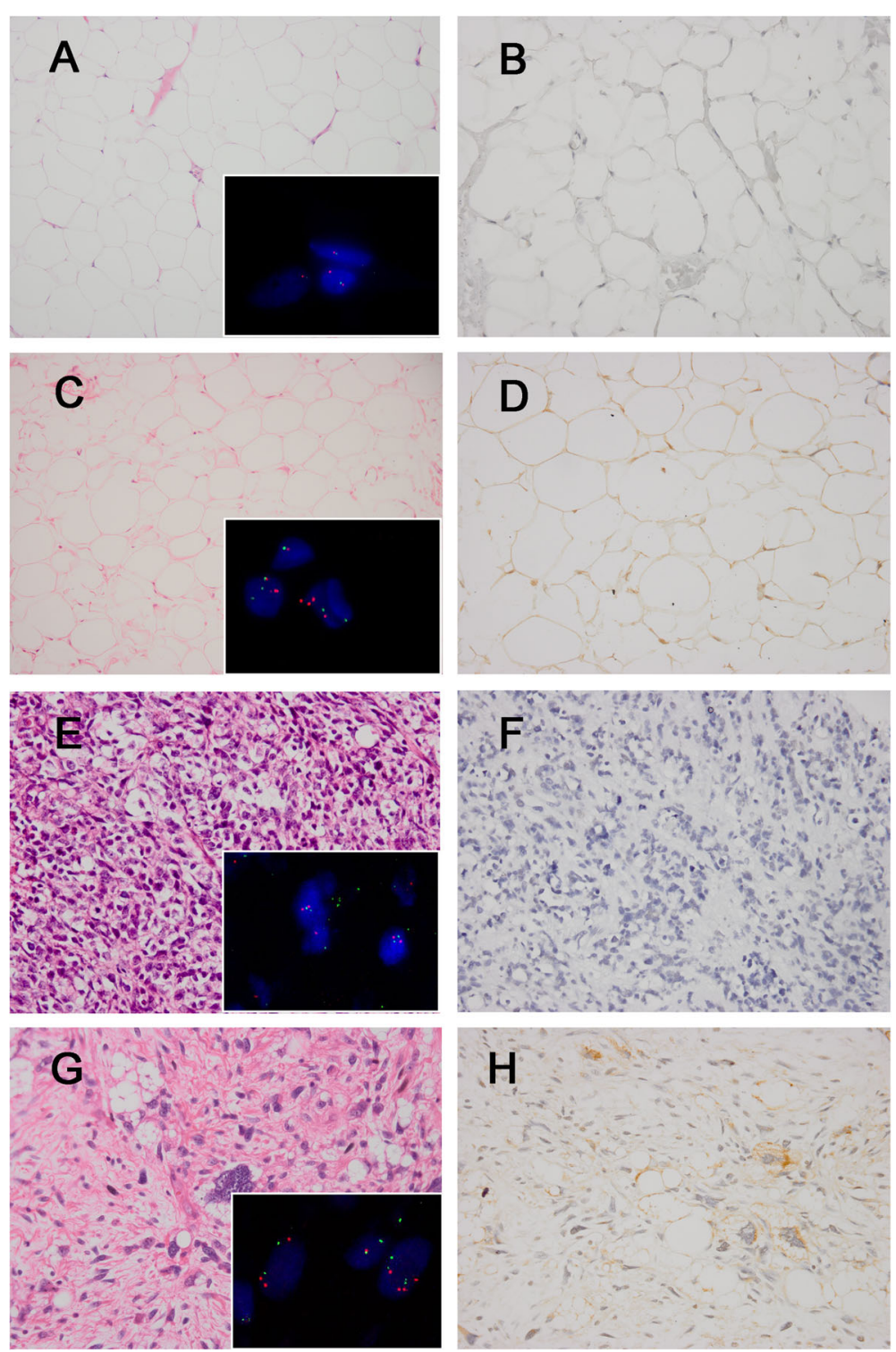

Fig. 3 The histologic features of control tumors and corresponding FRS2 FISH and FRS2 immunohistochemical staining results. Lipoma(a) without FRS2 gene amplification (inset) was FRS2 immunostaining negative (b). Lipoma(c) without FRS2 gene amplification (inset) but exhibited $1+$ FRS2 expression (d). Pleomorphic liposarcoma(e) was FRS2 nonamplified (inset) and negative for FRS2 immunostaining (f). Pleomorphic liposarcoma(g) without FRS2 amplification showing polysomic for CEP12 (inset), and had 1+ FRS2 staining (h). Red signals represent FRS2. Green signals represent chromosome12 centromeres. (a, c, e, g, haematoxylin and eosin; b, d, f, h, immunostaining) (original magnification, a, c × 200, $b, d-h \times 400)$

the consistent amplification of MDM2 and CDK4 in ALT/ WDL/DDL, FISH and IHC for the identification of $M D M 2$ and CDK4 amplification or overexpression have been widely used for the differential diagnosis of this entity [7-13]. Recently, the amplification of the FRS2 gene in ALT/WDL/DDL was identified by several studies, including our research group [14-16]. The use of FRS2 IHC was assessed by Zhang et al. in only 11 DDLs [15]. Here, we performed FRS2 IHC in 182 ALT/WDL/DDL and 64 histologic mimics with corresponding genetic data.
In the present study, 172 of 182 (94.5\%) ALT/WDL/ DDL showed FRS2 amplification by FISH, and 10 cases were FRS2-nonamplified but MDM2-amplified. The high frequency of FRS2 amplification of ALT/WDL/DDL in our research was similar to the results of 2 previous studies, with amplification frequencies of 100 and 93\%, respectively [14, 15]. Immunostaining results showed that 171 of $182(94.0 \%)$ ALT/WDL/DDL cases were positive for FRS2, including 83 ALT/WDLs and 88 DDLs. The positivity of FRS2 IHC (94.0\%) was similar to 
Table 2 FRS2 immunostaining and FISH results in 91ALT/WDLs and 91 DDLs

\begin{tabular}{|c|c|c|c|c|c|}
\hline \multirow[t]{2}{*}{ FRS2 FISH } & \multicolumn{4}{|c|}{ FRS2 immunostaining } & \multirow[t]{2}{*}{ Tota } \\
\hline & $-(\%)$ & $1+(\%)$ & $2+(\%)$ & $3+(\%)$ & \\
\hline FRS2 amplified & $6(3.5 \%)$ & $46(26.7 \%)$ & $63(36.6 \%)$ & $57(33.2 \%)$ & 172 \\
\hline ALT/WDL & $3(3.6 \%)$ & $31(36.9 \%)$ & $31(36.9 \%)$ & $19(22.6 \%)$ & 84 \\
\hline DDL & $3(3.4 \%)$ & $15(17.0 \%)$ & $32(36.4 \%)$ & $38(43.2 \%)$ & 88 \\
\hline FRS2 non-amplified & $5(50 \%)$ & $5(50 \%)$ & $0(0 \%)$ & $0(0 \%)$ & 10 \\
\hline ALT/WDL & $5(71.4 \%)$ & $2(28.6 \%)$ & $0(0 \%)$ & $0(0 \%)$ & 7 \\
\hline DDL & $0(0 \%)$ & $3(100 \%)$ & $0(0 \%)$ & $0(0 \%)$ & 3 \\
\hline
\end{tabular}

ALT/WDL Atypical lipomatous tumor/well-differentiated liposarcoma, DDL Dedifferentiated liposarcoma, FISH fluorescence in situ hybridization, - negativity, 1+ weak positivity, $2+$ moderate positivity, $3+$ strong positivity

the positivity of FRS2 FISH (94.5\%) in 182 cases, with relatively good consistency $(P<0.05$, kappa $=0.444)$. In 172 FRS2-amplified cases, $166(96.5 \%)$ cases were positive for FRS2 IHC, including 120 tumors (69.8\%) demonstrating $2 / 3+$ positivity. The high positive rate of FRS2 immunostaining in ALT/WDL/DDL indicated that FRS2 IHC could be a screening tool for ALT/WDL/DDL cases. It should be mentioned that 6 FRS2-amplified cases were FRS2-immunonegative, consisting of 3 ALT/ WDLs and 3 DDLs. In the 6 FRS2-immunonegative cases, effect of storage time and tumor necrosis or hemorrhage of some samples (5/6) may be related to false negative results. In 10 FRS2-nonamplified cases, 5 cases were also FRS2-immunonegative, while 5 cases showed 1+ FRS2 positivity, including 2 ALT/WDLs and 3 DDLs. The necrotic area and hemorrhagic area were identified in 3 of the 5 FRS2-immunopositive cases, which might be associated with false positive results.

In 64 FRS2-nonamplified control cases, 47 (73.5\%) cases were negative for FRS2 immunostaining and 17 were FRS2-immunopositive, including 2 cases with $2+$ positivity and 15 cases with $1+$ positivity. It should be mentioned that the FISH analysis of the pleomorphic liposarcoma with $2+$ positivity showed polysomic for CEP12, which may lead to FRS2-immunopositivity in this case. Moreover, among the 15 cases with 1+ FRS2 expression, 2 tumors also harbored a polysomic pattern (1 pleomorphic liposarcoma and 1 undifferentiated pleomorphic sarcoma). Other changes such as necrosis or hemorrhage were found in 5 cases (2 leiomyosarcomas,
1 pleomorphic liposarcoma, 1 lipoma and 1 spindle cell lipoma). Therefore, the evaluation should be careful in the cases exhibiting $1+$ positivity with hemorrhage and/ or necrosis.

This series showed that the diagnostic sensitivity and specificity of FRS2 IHC were 94.0 and $73.5 \%$, respectively, in distinguishing ALT/WDL/DDL from other histological mimics. The results suggested that FRS2 could be used as a screening tool for ALT/WDL/DDL. In addition, we analyzed the utility of FRS2 IHC for differentiating ALT/WDL and DDL from their own morphological simulators. When we used $1+$ or $2+$ as the threshold value, the sensitivity and specificity of FRS2 to distinguish ALT/WDL from fat tissue and benign adipocytic tumors were 91.2 and $80.0 \%, 54.9$ and $100 \%$, respectively. In differentiating DDL from other spindle cell tumors, the sensitivity and specificity of FRS2 were 96.7 and $65.5 \%$, respectively. Interestingly, when using $2+$ staining as the cutoff value in the differentiation of DDL with its histologic mimic, the sensitivity and specificity of FRS2 IHC changed to 76.9 and $93.1 \%$, respectively. Hence, when one case had 2+ FRS2 immunostaining positivity, ALT/WDL/DDL should be highly suspected.

The total positive rate of FRS2 immunostaining in our cohort was generally similar to that of existing diagnostic markers (MDM2 and CDK4). In our series, the sensitivity of FRS2 immunostaining seemed to be slightly lower than that of MDM2 (FRS2 vs. MDM2: $94.0 \%$ vs 100.0\%) and CDK4 (FRS2 vs. CDK4: $94.0 \%$ vs 97.0\%), whereas the sensitivities of MDM2 and CDK4 in previous studies

Table 3 Sensitivity and specificity of FRS2 immunostaining in diagnosing ALT/WDLs and DDLs

\begin{tabular}{|c|c|c|c|c|}
\hline \multirow[t]{3}{*}{ Tumor type } & \multicolumn{4}{|l|}{ FRS2 } \\
\hline & \multicolumn{2}{|l|}{$\geq 1+$} & \multicolumn{2}{|l|}{$\geq 2+$} \\
\hline & Sensitivity & Specificity & Sensitivity & Specificity \\
\hline ALT/WDL/DDL VS. control group & $94.0 \%$ & $73.5 \%$ & $66.0 \%$ & $96.9 \%$ \\
\hline ALT/WDL VS. BAT \& normal adipose tissue & $91.2 \%$ & $80.0 \%$ & $54.9 \%$ & $100 \%$ \\
\hline DDL VS. other spindle cell tumors & $96.7 \%$ & $65.5 \%$ & $76.9 \%$ & $93.1 \%$ \\
\hline
\end{tabular}

ALT/WDL Atypical lipomatous tumor/well-differentiated liposarcoma, DDL Dedifferentiated liposarcoma, BAT benign adipocytic tumor, $1+$ weak positivity, $2+$ moderate positivity; 

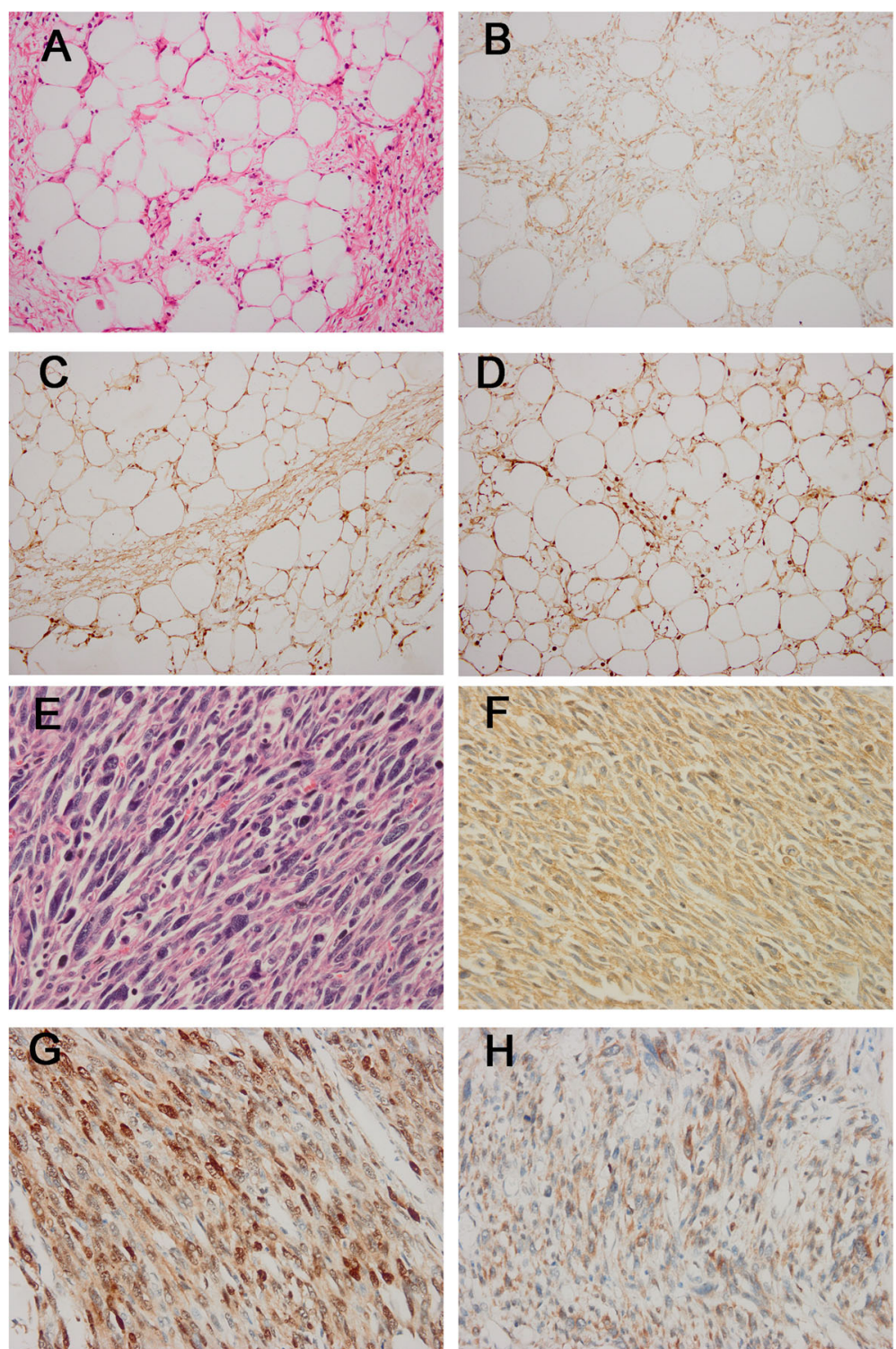

Fig. 4 The histologic features of ALT/WDL and DDL and the corresponding FRS2, MDM2 and CDK4 immunohistochemical staining results. ALT/ WDL (a) exhibited strong FRS2 immunostaining (b) and showed positive MDM2 (c) and CDK4 (d) immunostaining results. DDL(e) had strong FRS2 (f) immunostaining and positive MDM2 (g) and CDK4 (h) immunostaining results. (a, e, haematoxylin and eosin; b-d,f-h, immunostaining) (original magnification, a-d $\times 200$, e- $h \times 400$ )

ranged from 45 to $100 \%$ and 41 to $100 \%$, respectively [ 9 , $11,12,21-23]$. It should be emphasized that MDM2 and CDK4 also could show false positivity in the MDM2nonamplified cases and the specificity of MDM2 and CDK4 immunostaining ranged from 60 to $100 \%$ in different studies [11, 12, 21, 23-28]. It should be noted that in this study the specificity of FRS2 immunostaining was slightly higher than the result of MDM2 (FRS2 vs. MDM2: $73.5 \%$ vs $67.8 \%$ ) and CDK4 (FRS2 vs. CDK4: $73.5 \%$ vs 64.4\%). Therefore, FRS2, MDM2 and CDK4 may be complementary in the diagnosis of ALT/WDL/
DDL. Notably, molecular analysis should be considered for challenging cases.

The main therapeutic strategy for ALT/WDL/DDL is surgical resection; in the meantime, targeted therapy has been developed and is worthy of further exploration, especially for unresectable tumors. In recent years, MDM2 antagonists and CDK4 inhibitors have been used in clinical and preclinical studies, and some of them obtained favorable outcomes [29-32]. In our study, the analysis of FRS2 gene amplification and expression can help in the diagnosis of ALT/WDL/DDL. Moreover, as the key 
adaptor of the FGFR pathway, the overexpression of FRS2 in ALT/WDL/DDL indicated that it could play an important role in the targeted therapy of this disease. Zhang et al. identified activation of FGFR/FRS2 pathways in liposarcoma, and the use of FGFR inhibitors could significantly inhibit the growth of liposarcoma cells. Other groups also reported that FGFR inhibitors could inhibit cell proliferation in FRS2-amplified DDL cell lines, further demonstrating the possible therapeutic use of the FGFR/FRS2 pathway in ALT/WDL/DDL [15, 33, 34]. In addition, recent studies showed that pharmacologically targeting FRS2 inhibited FGF/FGFR-mediated oncogenic signaling and tumor progression in prostate cancer and gastric cancer cell lines [35]. Further investigations are warranted to further evaluate the therapeutic prospects of the FGFR/FRS2 pathway in ALT/WDL/ DDL.

In summary, our study analyzed the expression of FRS2 protein in $182 \mathrm{ALT} / \mathrm{WDL} / \mathrm{DDL}$ cases and compared the result with genetic data. The FRS2 immunostaining had relatively good consistency with FRS2 FISH. Moreover, FRS2 immunostaining had a slightly lower sensitivity but a slightly higher specificity than that of classic IHC markers (MDM2 and CDK4). It also should be noted that FRS2 IHC performed in a similar fashion to MDM2 and CDK4, which are also imperfect in their way with a subset of cases showing false immunopositivity in $M D M 2 / C D K 4 / F R S 2$-nonamplified samples. This new marker could be utilized as an additional screening tool for the diagnosis of ALT/WDL/DDLs in laboratories without the possibility of performing in situ hybridization assays. Importantly, careful histological inspection, immunohistochemical and molecular tests can help to arrive at correct diagnosis for challenging cases. It must be emphasized that $M D M 2 / C D K 4 / F R S 2$ especially $M D M 2$ FISH remains the gold standard and the most recommended method to diagnose this entity. In the future, the amplification and overexpression of the FRS2 gene in this disease spectrum might provide new clues for the targeted therapy of ALT/WDL/DDL.

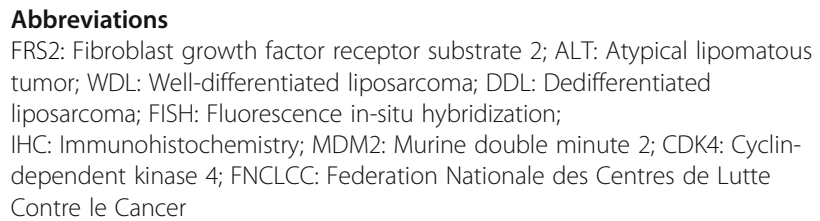

FRS2: Fibroblast growth factor receptor substrate 2; ALT: Atypical lipomatous tumor; WDL: Well-differentiated liposarcoma; DDL: Dedifferentiated liposarcoma; FISH: Fluorescence in-situ hybridization;

IHC: Immunohistochemistry; MDM2: Murine double minute 2; CDK4: Cyclindependent kinase 4; FNCLCC: Federation Nationale des Centres de Lutte Contre le Cancer

\section{Acknowledgements}

Not applicable.

\section{Authors' contributions}

Wenyi Jing: data analysis, test performance and writing. Ting Lan: data analysis, test performance. Yan Qiu and Ran Peng: data analysis and collect information. Yang Lu and Huijiao Chen: data review and histopathological examinations. Min Chen: help molecular experiments. Xin He and Chen Chen: review of histopathology. Hongying Zhang: the corresponding author, study design, and the manuscript revision. All authors read and approved the final manuscript.

\section{Funding}

This work was supported by the National Natural Science Foundation of China (No. 81972520, 81472510), Sichuan Science and Technology Program (NO.2020YFS0270), and the 135 Project for Disciplines of Excellence-Clinical Research Incubation Project, West China Hospital, Sichuan University (NO.2018HXFH011).

\section{Availability of data and materials}

All data generated or analysed during this study are included in this published article and its supplementary information files.

\section{Declarations}

Ethics approval and consent to participate

This study was approved by the West China Hospital Institutional Review Board.

Consent for publication

Not applicable.

\section{Competing interests}

The authors declare that they have no competing interests.

\section{Author details}

1Department of Pathology, West China Hospital, Sichuan University, Guoxuexiang 37, Chengdu 610041, Sichuan, China. ${ }^{2}$ Department of Pathology, Sichuan Cancer Hospital \& Institute, Sichuan Cancer Center, Cancer Hospital affiliate to School of Medicine, University of Electronic Science and Technology of China, Chengdu, China.

Received: 12 June 2021 Accepted: 6 October 2021

Published online: 25 October 2021

\section{References}

1. Sbaraglia M, Dei Tos AP, Pedeutour F. Atypical lipomatous tumour/welldifferentiated liposarcoma. In: WHO classification of Tumours editorial board, editor. WHO Classification of Tumours of Soft Tissue and Bone. 5th ed. Lyon: IARC Press; 2020. p. 36-8.

2. Dei Tos AP, Marino-Enriquez A, Pedeutour F. Dedifferentiated liposarcoma. In: WHO classification of Tumours editorial board, editor. WHO Classification of Tumours of Soft Tissue and Bone. 5th ed. Lyon: IARC Press; 2020. p. 3941.

3. Goldblum JR, Folpe AL, Weiss SW. Liposarcoma. In: Goldblum JR, Folpe AL, Weiss SW, editors. Enzinger and Weiss's soft tissue tumors. 6th ed. Philadelphia: Saunders/Elsevier; 2014. p. 484-523.

4. Sandberg AA. Updates on the cytogenetics and molecular genetics of bone and soft tissue tumors: liposarcoma. Cancer Genet Cytogenet. 2004;155:124

5. Italiano A, Bianchini L, Keslair F, et al. HMGA2 is the partner of MDM2 in well-differentiated and dedifferentiated liposarcomas whereas CDK4 belongs to a distinct inconsistent amplicon. Int J Cancer. 2008;122:2233-41.

6. Erickson-Johnson MR, Seys AR, Roth CW, et al. Carboxypeptidase M: a biomarker for the discrimination of well-differentiated liposarcoma from lipoma. Mod Pathol. 2009:22:1541-7.

7. Weaver J, Downs-Kelly E, Goldblum JR, et al. Fluorescence in situ hybridization for MDM2 gene amplification as a diagnostic tool in lipomatous neoplasms. Mod Pathol. 2008;21:943-9.

8. Zhang $\mathrm{H}$, Erickson-Johnson M, Wang $\mathrm{X}$, et al. Molecular testing for lipomatous tumors: critical analysis and test recommendations based on the analysis of 405 extremity-based tumors. Am J Surg Pathol. 2010;34: 1304-11.

9. Sirvent N, Coindre JM, Maire G, et al. Detection of MDM2-CDK4 amplification by fluorescence in situ hybridization in 200 paraffinembedded tumor samples: utility in diagnosing adipocytic lesions and comparison with immunohistochemistry and real-time PCR. Am J Surg Pathol. 2007;31:1476-89.

10. Binh MB, Sastre-Garau X, Guillou L, et al. MDM2 and CDK4 immunostainings are useful adjuncts in diagnosing well-differentiated and dedifferentiated 
liposarcoma subtypes: a comparative analysis of 559 soft tissue neoplasms with genetic data. Am J Surg Pathol. 2005;29:1340-7.

11. Aleixo PB, Hartmann AA, Menezes IC, et al. Can MDM2 and CDK4 make the diagnosis of well differentiated/dedifferentiated liposarcoma? An immunohistochemical study on 129 soft tissue tumours. J Clin Pathol. 2009; 62:1127-35.

12. Thway K, Flora R, Shah C, et al. Diagnostic utility of p16, CDK4, and MDM2 as an immunohistochemical panel in distinguishing well-differentiated and dedifferentiated liposarcomas from other adipocytic tumors. Am J Surg Pathol. 2012;36:462-9.

13. Kammerer-Jacquet SF, Thierry S, Cabillic F, et al. Differential diagnosis of atypical lipomatous tumor/well-differentiated liposarcoma and dedifferentiated liposarcoma: utility of p16 in combination with MDM2 and CDK4 immunohistochemistry. Hum Pathol. 2017;59:34-40.

14. Wang X, Asmann YW, Erickson-Johnson MR, et al. High-resolution genomic mapping reveals consistent amplification of the fibroblast growth factor receptor substrate 2 gene in well-differentiated and dedifferentiated liposarcoma. Genes Chromosom Cancer. 2011;50:849-58.

15. Zhang K, Chu K, Wu X, et al. Amplification of FRS2 and activation of FGFR/ FRS2 signaling pathway in high-grade liposarcoma. Cancer Res. 2013;73: 1298-307.

16. Jing W, Lan T, Chen H, et al. Amplification of FRS2 in atypical lipomatous tumour/well-differentiated liposarcoma and de-differentiated liposarcoma: a clinicopathological and genetic study of 146 cases. Histopathology. 2018;72: $1145-55$.

17. Turner N, Grose R. Fibroblast growth factor signalling: from development to cancer. Nat Rev Cancer. 2010;10:116-29.

18. Coindre JM. Grading of soft tissue sarcomas: review and update. Arch Pathol Lab Med. 2006;130:1448-53.

19. He X, Pang Z, Zhang X, et al. Consistent amplification of FRS2 and MDM2 in low-grade osteosarcoma: a genetic study of 22 cases with Clinicopathologic analysis. Am J Surg Pathol. 2018:42:1143-55.

20. Li Z, Huang C, Bai S, et al. Prognostic evaluation of epidermal fatty acidbinding protein and calcyphosine, two proteins implicated in endometrial cancer using a proteomic approach. Int J Cancer. 2008;123:2377-83.

21. Dei Tos AP, Doglioni C, Piccinin S, et al. Coordinated expression and amplification of the MDM2, CDK4, and HMGI-C genes in atypical lipomatous tumours. J Pathol. 2000;190:531-6.

22. Clay MR, Martinez AP, Weiss SW, et al. MDM2 and CDK4 immunohistochemistry: should it be used in problematic differentiated Lipomatous tumors?: a new perspective. Am J Surg Pathol. 2016;40:1647-52.

23. Brimo F, Dion D, Huwait $H$, et al. The utility of MDM2 and CDK4 immunohistochemistry in needle biopsy interpretation of lipomatous tumours: a study of 21 Tru-cut biopsy cases. Histopathology. 2008:52:892-5.

24. Pilotti S, Della Torre G, Mezzelani A, et al. The expression of MDM2/CDK4 gene product in the differential diagnosis of well differentiated liposarcoma and large deep-seated lipoma. Br J Cancer. 2000;82:1271-5.

25. Hung YP, Michal M, Dubuc AM, et al. Dysplastic lipoma: potential diagnostic pitfall of using MDM2 RNA in situ hybridization to distinguish between lipoma and atypical lipomatous tumor. Hum Pathol. 2020;101:53-7.

26. Kobayashi A, Sakuma T, Fujimoto M, et al. Diagnostic utility and limitations of immunohistochemistry of p16, CDK4, and MDM2 and automated dualcolor in situ hybridization of MDM2 for the diagnosis of challenging cases of dedifferentiated Liposarcoma. Appl Immunohistochem Mol Morphol. 2019;27:758-63.

27. Anderson WJ, Fletcher CDM, Jo VY. Atypical pleomorphic Lipomatous tumor: expanding our current understanding in a Clinicopathologic analysis of 64 cases. Am J Surg Pathol. 2021. https://doi.org/10.1097/PAS. 0000000000001706.

28. Alshenawy H. Can HMGI-C be used as an aid with MDM2 and CDK4 to differentiate liposarcoma subtypes from their mimics? J Cancer Res Clin Oncol. 2013;139:1073-81.

29. Ray-Coquard I, Blay JY, Italiano A, et al. Effect of the MDM2 antagonist RG7112 on the P53 pathway in patients with MDM2-amplified, welldifferentiated or dedifferentiated liposarcoma: an exploratory proof-ofmechanism study. Lancet Oncol. 2012;13:1133-40.

30. Bill KL, Garnett J, Meaux I, et al. SAR405838: a novel and potent inhibitor of the MDM2:p53 Axis for the treatment of dedifferentiated Liposarcoma. Clin Cancer Res. 2016;22:1150-60.
31. Dickson MA, Tap WD, Keohan ML, et al. Phase II trial of the CDK4 inhibitor PD0332991 in patients with advanced CDK4-amplified well-differentiated or dedifferentiated liposarcoma. J Clin Oncol. 2013;31:2024-8.

32. Dickson MA, Schwartz GK, Keohan ML, et al. Progression-free survival among patients with well-differentiated or dedifferentiated Liposarcoma treated with CDK4 inhibitor Palbociclib: a phase 2 clinical trial. JAMA Oncol. 2016:2:937-40

33. Hanes R, Grad I, Lorenz $\mathrm{S}$, et al. Preclinical evaluation of potential therapeutic targets in dedifferentiated liposarcoma. Oncotarget. 2016;7: 54583-95.

34. Hanes $\mathrm{R}$, Munthe $\mathrm{E}$, Grad I, et al. Preclinical evaluation of the Pan-FGFR inhibitor LY2874455 in FRS2-amplified Liposarcoma. Cells. 2019;8:189.

35. Li Q, Alsaidan OA, Ma Y, et al. Pharmacologically targeting the myristoylation of the scaffold protein FRS2a inhibits FGF/FGFR-mediated oncogenic signaling and tumor progression. J Biol Chem. 2018;293:6434-48.

\section{Publisher's Note}

Springer Nature remains neutral with regard to jurisdictional claims in published maps and institutional affiliations.

\section{Ready to submit your research? Choose BMC and benefit from:}

- fast, convenient online submission

- thorough peer review by experienced researchers in your field

- rapid publication on acceptance

- support for research data, including large and complex data types

- gold Open Access which fosters wider collaboration and increased citations

- maximum visibility for your research: over $100 \mathrm{M}$ website views per year

At BMC, research is always in progress.

Learn more biomedcentral.com/submissions 